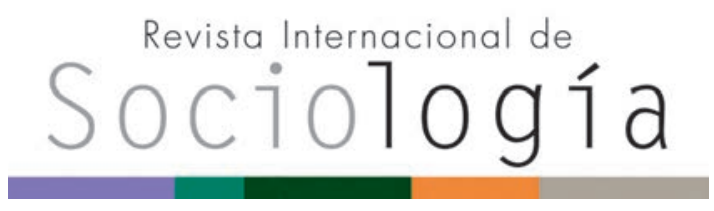

Revista Internacional de Sociología RIS

vol. 77 (2), e129, abril-junio, 2019, ISSN-L:0034-9712 https://doi.org/10.3989/ris.2019.77.2.18.052

\section{EL PAPEL DE LAS REDES FAMILIARES EN LAS DECISIONES DE INMOVILIDAD ESPACIAL EN EL ÁREA METROPOLITANA DE GRANADA}

\author{
ISABEL PALOMARES LINARES \\ Universidad de Granada \\ ipalomares@ugr.es
}

ORCID iD: https://orcid.org/0000-0001-6795-2987

\author{
Ricardo Duque CALVACHE \\ Universidad de Granada \\ ricardoduque@ugr.es \\ ORCID iD: https://orcid.org/0000-0002-6356-5071 \\ JOAQUín SUSINO ARBUCIAS \\ Universidad de Granada \\ jsusino@ugr.es \\ ORCID iD: https://orcid.org/0000-0001-9096-6172
}

Cómo citar este artículo / Citation: Palomares Linares, I., R. Duque Calvache y J. Susino Arbucias. 2019. "El papel de las redes familiares en las decisiones de inmovilidad espacial en el área metropolitana de Granada". Revista Internacional de Sociología 77(2):e129. https:// doi.org/10.3989/ris.2019.77.2.18.052

\section{RESUMEN}

Pese a que la familia es una reconocida institución con influencia en múltiples facetas de la vida social en España, no existen muchos estudios específicos sobre su papel en las trayectorias residenciales. Utilizando una encuesta realizada en el área metropolitana de Granada en 2008, en este trabajo analizamos la influencia de dos aspectos - la concentración de la red familiar y la presencia de raíces en el barrio- en las decisiones de movilidad e inmovilidad espacial. Los resultados indican que las redes familiares juegan un papel importante para entender las decisiones de sedentarismo espacial en nuestro contexto de estudio. La familia es un fuerte factor de arraigo en la vivienda y en el barrio de toda la población, pero, al mismo tiempo, está modulado por la posición social de los individuos y las familias y su importancia es mayor entre las familias con menores recursos y para aquellos que residen en barrios más desfavorecidos.

\section{Palabras Clave}

Barrio; (In)movilidad residencial; Raíces familiares; Redes familiares.

\section{THE ROLE OF FAMILY \\ NETWORKS IN THE SPATIAL IMMOBILITY DECISIONS IN THE METROPOLITAN AREA OF GRANADA}

Copyright: (C) 2019 CSIC. Este es un artículo de acceso abierto distribuido bajo los términos de la licencia de uso y distribución Creative Commons Reconocimiento 4.0 Internacional (CC BY 4.0).

Recibido: 01/04/2018. Aceptado: 28/09/2018 Publicado online: 04/06/2019

\section{Abstract}

Even though family is acknowledged to be a key institution is Spain, with a deep influence in multiple facts of social life, few studies have analyzed its role on the individuals' residential trajectories. Using data from a 2008 local survey conducted in the metropolitan area of Granada, this paper researches the importance of two factors- concentration of the family network, and presence of family roots- in spatial mobility and seden-tarism. Our results underline how family networks are crucial to understand why people stay in our context. It is a strong reason to stay in the house and the neigh-borhood for everyone, but at the same time is modified by the social position of the individuals and the families, having a stronger effect on low income families and dis-advantaged neighbourhoods.

\section{KEYWORDS}

Family networks; Family roots; Neighborhood; Residen-tial (in)mobility. 


\section{INTRODUCCIÓN}

En España, al igual que en otros países mediterráneos, la familia es una institución con un rol relevante en múltiples facetas de la vida social (De Pablos y Susino 2010; Flaquer 2004). Desde una perspectiva europea, es fundamental tener en cuenta la importancia de los lazos familiares en los países del sur (Jordan 1988) o las actitudes y normas sociales diferenciales sobre las funciones y obligaciones del grupo familiar con respecto a sus miembros (Reher 1998; Tomassini et al. 2004). Sin embargo, pese a la relevancia de lo familiar en países mediterráneos, son pocos los trabajos sobre su influencia en las decisiones residenciales de sus miembros (Módenes Cabrerizo 1998; Alabart 2007). Sobre todo, cuando dichas decisiones se refieren a los cambios en un mismo espacio de vida metropolitano y no conllevan un movimiento migratorio. Aún sabemos poco acerca de quiénes viven cerca de su familia ni de cómo está conectada la geografía urbana de la red familiar con las decisiones de movilidad espacial.

La mayor parte de las investigaciones realizadas al respecto se refieren a la proximidad entre padres e hijos, con datos a nivel nacional (Hank 2007; Rainer y Siedler 2012). Los resultados identifican factores macroestructurales que influyen en el comportamiento residencial de los miembros de una misma familia (sobre todo, cuando alguno de ellos tiene una mayor necesidad de ayuda). En España, al igual que en otros países mediterráneos, la proximidad residencial y la corresidencia entre generaciones es más frecuente que en el norte de Europa. Pero, aunque dichas comparaciones en el nivel macro son necesarias, si se quiere comprender en qué medida los factores micro marcan diferencias entre los diversos actores y grupos sociales es necesario descender al nivel de estudio de cada ciudad metropolitana como marco en que cobran sentido las decisiones de movilidad. El objetivo de este trabajo es conocer cuál es la relación entre redes familiares y comportamiento residencial en el área metropolitana de Granada. Nos interesa descubrir quiénes tienen una mayor probabilidad de vivir cerca de su familia; y conocer cómo la red familiar próxima puede estar motivando la movilidad o la permanencia en el domicilio y el barrio.

La relación entre familia y movilidad es, con certeza, compleja y requiere un cuidadoso análisis (Cooke 2008; Mulder y Malmberg 2014; Mulder y Cooke 2009; Spring et al. 2017). Utilizando una encuesta sobre población y vivienda realizada en 2008, nos centramos en dos variables concretas: la presencia de raíces familiares y el grado de concentración de la red familiar en el barrio. Dada la limitada información disponible a nivel nacional y local para tratar dichas cuestiones, consideramos que es una fuente muy adecuada para conectar el comportamiento residencial, las redes familiares y su localización en el espacio urbano.

\section{LOCALIZACIÓN DE REDES FAMILIARES E (IN)MOVILIDAD RESIDENCIAL}

La proximidad residencial entre parientes puede ser entendida como una consecuencia de las decisiones migratorias (Hank 2007). Pero también puede ser entendida como factor relevante en el proceso en la toma de decisiones (Mulder 2007), esto es, como causante de las mismas. En esta línea y desde una perspectiva holística y relacional, podemos entender que las prácticas de (in)movilidad están configuradas por las circunstancias y trayectorias del hogar, pero también están conectadas con las geografías de nuestras redes sociales y familiares y con las estructuras y contextos socioespaciales donde dichas conexiones se producen y reproducen (Coulter, Van Ham y Findlay 2015). La red familiar fuera del hogar puede por tanto desempeñar un papel en cuándo o dónde moverse (Michielin, Mulder y Zorlu 2008; Pettersson y Malmberg 2009) así como en cuándo y dónde quedarse (Fischer y Malmberg 2001; Dawkins 2006; Clark, Duque-Calvache y Palomares-Linares 2015).

Un gran parte de las investigaciones se ha centrado en conocer en qué circunstancias los individuos y los hogares se mueven cerca de sus familiares en relación con los perfiles y características de los parientes a los que se acercan. Dada la relevancia que adquiere el estudio de los lazos intergeneracionales, muchos investigadores han dado prioridad al análisis de los movimientos de proximidad entre padres e hijos. Sabemos que las situaciones de dependencia funcional de algún pariente cercano o acontecimientos en el curso vital, como la llegada del primer hijo o las rupturas conyugales, pueden desencadenar movimientos de proximidad de los hijos con respecto al domicilio de los padres, pero también de los padres con respecto a los hijos (Rossi y Rossi 1990; Michielin y Mulder 2008; Pettersson y Malmberg 2009). Como Smits (2010) apunta, en general, es más probable que las generaciones busquen un acercamiento cuando se produce un incremente en la necesidad de ayuda en cualquiera de los dos hogares, aunque la persona o el hogar que presenta la necesidad es la que suele protagonizar el movimiento.

De forma opuesta, se han señalado también factores que hacen más probable quedarse. Por ejemplo, los años transcurridos desde el anterior cambio, la fuerza de las redes sociales tejidas, tener hijos escolarizados en el entorno próximo o la convivencia en pareja - respecto a vivir solo- pueden producir una mayor ligazón al territorio. Por supuesto, los acontecimientos en el curso vital no son ajenos a las condiciones de los sujetos que los viven. El género y su asociación a roles de cuidado (Joseph y Hallman 1998), la posición socioeconómica (Clark y Wolf 1992; Spilimbergo y Ubeda 2004), la etnia (Crowder y South 2005; Zorlu y Latten 2009) o la educación (Hank 2007; López-Gay 2016) son variables moduladoras de las anteriormente mencionadas y que, además, marcan diferencias significativas de por sí. En este sentido, parece poco cues- 
tionable que la medida en que un hogar o individuo requiere ayuda y en qué medida no puede obtenerla por otros medios son cuestiones inseparables a la hora de entender por qué las personas se mueven o se quedan cerca de su anterior hogar (Módenes Cabrerizo 1998).

Últimamente, al estudio de la movilidad de proximidad entre padres e hijos se le suman artículos cuyo interés se centra en saber las implicaciones residenciales de otras formas de parentesco (entre abuelos y nietos en Kolk, 2016; entre hermanos y familia extensa en Hedman, 2013). De igual forma, se empieza a analizar no solo los perfiles de los que se mueven, sino también la importancia que la presencia de la red familiar tiene en la elección de destino. Hedman (2013), pone de manifiesto que, cuando analizamos la movilidad dentro de una misma ciudad, la localización de parientes puede estar jugando un papel en las elecciones que los móviles hacen entre los distintos barrios. En todo caso, lo que reconocen estos nuevos enfoques es que: (a) los efectos que ejerce la red familiar en la movilidad no se limitan a la influencia intergeneracional nuclear entre padres e hijos; (b) si queremos avanzar en el conocimiento de cómo están asociadas las redes familiares y las decisiones residenciales, debemos contextualizar nuestros análisis de formas más significativas (por ejemplo, analizando su influencia en barrios o ciudades concretas).

\section{FAMILIA Y COMPORTAMIENTO RESI- DENCIAL EN ESPAÑA}

Hank (2007), en un estudio comparativo entre países europeos, descubre que los patrones de contacto y de proximidad residencial entre hijos y padres son muy diferentes regionalmente. En España, la probabilidad de que las generaciones vivan próximas y mantengan contactos intensos - más de una vez a la semana- es más alta que en países del norte, pero es parecida a la probabilidad encontrada en otros contextos mediterráneos -Italia, Grecia-. En la misma línea, Rainer y Siedler (2012) indican que en dichos contextos mediterráneos, en los que la familia es crucial en la provisión de cuidados a sus miembros más mayores, existe una mayor correlación entre proximidad residencial entre parientes que en los países donde la provisión está organizada y dotada de medios a través de otras instituciones públicas o privadas.

De inicio, dado que aceptamos la hipótesis de que la importancia de la familia es generalizada, podría pensarse que no encontraremos grandes diferencias al analizar qué individuos y qué hogares son más propensos a vivir cerca de la familia, sino que este recurso es transversal a todo tipo de familias. Pero no todos los grupos sociales o individuos a lo largo de su vida tienen las mismas necesidades de apoyo ni las mismas oportunidades para recibir la ayuda fuera de la red familiar (Van Groenou y Van Tilburg 2003). En primer lugar, los grupos sociales con menos recursos pueden ser más dependientes de las redes informales -y entendemos la familia como tal- para el desarrollo de su vida cotidiana (Litwak y Szelenyi 1969). Se asume un trade-off que implica que a menos recursos formales, más necesidad de recursos informales (Van Groenou y Van Tilburg 2003). Tal como Fors y Lennartsson (2008) apuntan, los individuos con menor estatus socioeconómico necesitan de la cercanía de sus familiares, aun cuando sus lazos de parentesco puedan ser ambivalentes o de naturaleza conflictiva (Kleinhans, van der Land y Doff 2010). En segundo lugar, los grupos sociales más desfavorecidos pueden haber "heredado" en gran medida esta condición de sus familias (van Ham et al. 2014). Tener que contribuir al mantenimiento de un hogar en situación precaria (ya sea económicamente o dedicando tiempo a tareas de apoyo o de cuidado) dificulta el acceso a mecanismos de movilidad social como los estudios o la movilidad geográfica. Por tanto, los miembros de una familia de escasos recursos pueden verse condicionados a la hora de cambiar de residencia, por lo que terminarían viviendo unos cerca de otros en mayor medida.

Las dos razones aportadas confluyen en una misma tendencia, marcada por la clase social y los recursos disponibles en la unidad familiar. Trabajos como el de Alabart (2007: 38) encuentran que los hogares en los extremos del espectro social —clases sociales más y menos vulnerables - muestran una marcada tendencia a buscar la proximidad familiar. Pero la amplia mayoría de la literatura alerta de que este patrón familista del comportamiento residencial es aún más acusado cuanto más bajamos en la escala social. Los hogares más pobres tenderán en mayor medida a vivir próximos a sus parientes, ya sea para recibir apoyo, para prestarlo o por pura incapacidad para salir de un entorno de privación (Palomares-Linares y van Ham 2018). Podemos incluso ampliar este efecto a nivel de barrio, asumiendo que en barrios más desfavorecidos aumentará la probabilidad de encontrar parientes en la proximidad (Preece 2018). Por tanto, nuestra primera hipótesis es que la probabilidad de vivir en el mismo barrio que los parientes es mayor entre los individuos con menores recursos y en barrios desfavorecidos.

\section{Orígenes famiLIARES Y CONCEN- TRACIÓN DE LA RED FAMILIAR EN EL BARRIO COMO PREDICTORES DE LA MOVILIDAD Y LA PERMANENCIA}

Cómo afecta a la vida de las personas haber crecido en un determinado espacio y en una determinada familia es un tema que levanta creciente interés en la academia y que destapa sugestivos resultados. Tener raíces en un lugar puede influir en las trayectorias espaciales que los individuos desarrollan una vez emancipados del hogar. Los miembros de una misma familia que se han socializado en un entorno deter- 
minado - urbano, suburbano o rural- pueden ser más propensos a escoger entornos similares una vez iniciadas sus carreras residenciales (Feijten, Hooimeijer y Mulder 2008), reproduciendo su propia historia familiar. La socialización temprana es un proceso que ocurre en espacios físicos y en esa etapa adoptamos también creencias sobre sobre los lugares que ocupamos (en relación con los que ocupan otros). Estas creencias pueden generar deseos y expectativas residenciales años después. Dado que los miembros de una misma familia tienen la misma socialización espacial, no es de extrañar que varios hermanos puedan escoger de manera independiente los mismos tipos de zonas e incluso que terminen residiendo en el mismo barrio una vez son adultos (Blaauboer 2011).

Por otra parte, haber crecido en un barrio asegura un grado de conocimiento de la zona, lo que se conoce como capital local específico (Da Vanzo 1981; Fischer y Malmberg 2001) y, por tanto, la percepción de mayores costes al cambiar de zona (Fors y Lennartsson 2008). Los años de vida pasados en un determinado lugar suele ser una de las variables fundamentales para entender por qué la gente se queda en el mismo (Clark y Dieleman 1996; Fischer y Malmberg 2001). Pero recientes estudios han demostrado que el conocimiento del barrio, haber vivido allí con anterioridad, puede ser también un factor con influencia en los movimientos de retorno (Hedman 2013; Blaauboer 2011). Aunque el retorno al lugar de origen es una de las claves en el estudio de la migración interior e internacional reciente en España (Puga 2004), los nuevos resultados advierten que las raíces familiares también pueden estar ejerciendo un papel en las trayectorias de (in)movilidad residencial, al menos en contextos urbanos europeos.

Los mecanismos por los cuales el grado de concentración de la red familiar en el barrio liga o atrae a otros parientes pueden generar una mayor controversia. En primer lugar, por el tipo de medida. En el análisis de redes sociales se utilizan indicadores relativos a la extensión y concentración de dichas redes (Campbell, Marsden y Hurlbert 1986; Thomése y Van Tilburg 2000). Pero también sabemos que dichos indicadores, en el estudio de las decisiones residenciales, pueden ser incompletos o generar resultados poco correctos si no se ponen en relación con su contexto. Querer moverse de un barrio puede venir motivado, no por la pobreza en el barrio per se, sino por la diferencia de nivel entre el individuo o familia y su barrio (van Ham y Feijten 2008).

Por otra parte, la calidad de las relaciones y su funcionalidad influyen más que la cantidad de contactos o personas que componen la red social cercana (Bell y Rutherford 2013). En definitiva, más extensión no es necesariamente sinónimo de mejor. Hay evidencia de que moverse cerca de los padres - 0 que los padres se trasladen cerca de los hijos- es más probable cuando también viven hermanos u otro tipo de parientes en los alrededores (Michielin y Mulder 2008). El atractivo de un lugar parece aumentar cuando distintos tipos de parientes viven allí (Dawkins 2006; Mulder y Van der Meer 2009).

Una segunda línea de debate se abre por la falta de consenso en cuáles son los efectos de una red más o menos densa (Fischer 1982; Granovetter 1973; Wellman y Berkowitz 1988). La mayor parte de los estudios se ha centrado en la densidad o concentración de las redes sociales en conjunto (no específicamente de las de tipo familiar), por lo que hay pocas y contradictorias evidencias.

Las redes familiares funcionan sobre todo como capital vinculante (bonding capital) y se caracterizan por generar fuertes vínculos entre sus miembros (Kleinhans 2009). La familia puede ser un recurso informativo o una plataforma de oportunidades, pero sobre todo es una fuente de recursos materiales, instrumentales y emocionales (Campbell, Marsden y Hurlbert 1986). En este sentido, redes familiares más concentradas pueden aportar más seguridad y jugar un papel esencial en facetas como la salud (Wood y Giles-Corti 2008) o en momentos críticos de la vida de sus miembros (Van Groenou y Van Tilburg 2003). Si la concentración de la red familiar en el barrio es muy alta, moverse al barrio o quedarse en él quizás sea la única forma de acceder a dichos recursos, sobre todo cuando el tipo de apoyo que se precisa o el tipo de lazo que se desea requiere una cierta cercanía espacial (Joseph y Hallman 1998).

Nuestra segunda hipótesis es que tener raíces familiares o una red familiar concentrada en el barrio aumenta la estabilidad residencial (tanto en la vivienda como en el barrio).

Bajo nuestro punto de vista, las raíces familiares y el grado de concentración juegan efectivamente un papel en las decisiones de los individuos como miembros de una red familiar, pero también se entrelazan en el proceso de reproducción social de las familias en el espacio y a lo largo del tiempo. Este doble efecto de atracción y fijación ha sido descrito en trabajos recientes (Spring et al. 2017). Residir en el mismo barrio que gran parte o toda la familia informa de la dimensión estructural, del grado o intensidad en que la reproducción social de la posición familiar puede estar ocurriendo. Pero, en este proceso, los individuos y las familias son actores entendibles desde la posición socioespacial que ocupan (Coulter, Van Ham y Findlay 2015). Y existe suficiente evidencia para sostener que las clases más vulnerables en los contextos más deprimidos son los que tienen oportunidades más limitadas para salir de su entorno (Fors y Lennartsson 2008).

Nuestra tercera hipótesis es que cuanto más baja es la clase social del individuo y del barrio en que se vive, mayor es la importancia de las variables familiares en la conducta residencial. 


\section{EL CONTEXTO ESPACIAL Y TEMPORAL: GRANADA EN EL COMIENZO DE LA CRISIS}

Si centramos nuestra atención en el caso de Granada, trabajamos con una ciudad de tamaño medio, pero con una forma y funcionamiento metropolitano (se acerca al medio millón de habitantes, aproximadamente la mitad de ellos en la capital y la mitad en la corona). Aunque el área incluye 46 municipios, su superficie urbana es bastante reducida: 137 kilómetros cuadrados en el año 2007 (Feria 2015). Estas características permiten que las familias puedan mantener el contacto con relativa facilidad aun residiendo en puntos lejanos del área. Esto introduce una diferencia con aquellas ciudades de mayor tamaño, en las cuales el coste temporal y económico de los desplazamientos puede multiplicarse, dificultando el contacto frecuente. Cabe, además, señalar que el proceso de suburbanización y expansión urbana ha sido protagonizado de manera mayoritaria por las clases trabajadoras (Susino 2003), lo que ha reforzado el carácter acomodado de la capital, una diferencia importante con respecto a otras ciudades en las que se ha producido una salida de las clases medias. Los grupos de rentas altas han tenido un comportamiento más sedentario de lo habitual, ocupando espacios que combinan la centralidad geográfica y la deseabilidad social debido, precisamente, a ese carácter burgués de sus habitantes. Por supuesto, esta situación dista de ser homogénea y podemos encontrar barrios en la capital con un carácter más obrero - casos de la Chana, el Zaidín o la zona norte- y también zonas de la corona donde son mayoritarias las clases medias, como en la zona sur, en los municipios situados en la falda de Sierra Nevada (Susino 2003).

Granada ha sido caracterizada como una ciudad donde la vida familiar juega un papel especialmente importante en la cotidianeidad de sus residentes, en cómo se relacionan y viven en el espacio (Palomares- Linares 2017) y también como elemento clave en la generación de apego residencial (Clark, Duque-Calvache y Palomares-Linares 2015). Por tanto, creemos que las conclusiones que alcancemos pueden ser extensibles para entender otros contextos en los que haya un acentuado familismo, característica compartida con, por ejemplo, las sociedades italiana o griega.

La influencia de los domicilios actuales y pasados de nuestros familiares en la forma en que nos movemos es un tema complejo y relevante. En los últimos años, debido a las consecuencias de la crisis, el foco de la atención se ha dirigido al creciente papel de la familia como mecanismo de apoyo, como un recurso adicional del que pueden disponer los hogares (Spring et al. 2017). Aunque no se han estudiado con precisión los efectos de este papel sobre la movilidad de corta distancia, es plausible suponer que la proximidad e incluso la cohabitación bajo el mismo techo (ya sean por elección o forzosas) han crecido en importancia desde el año 2008, en el que se realizó la encuesta en que basamos nuestros análisis. De hecho, aunque se suele considerar que el comienzo de la fase recesiva es anterior a dicho año, estimamos que los datos con los que trabajamos tampoco retratan la crisis. Tanto las cifras de empleo, que se desplomarán en años posteriores, como los ahorros acumulados en el periodo de bonanza (que fueron desapareciendo a medida que pasaban los años) indican que los datos recogidos en torno a 2011 — como los del Censo- capturan una situación sensiblemente más crítica.

Se han de tomar los resultados obtenidos como una medición de la importancia de los factores familiares en un contexto de crisis incipiente o, incluso, en el marco del final de la burbuja inmobiliaria. Durante el periodo estudiado, la ciudad continuaba su expansión territorial y la población de la corona iba aumentando frente a la residente en la capital. Asumimos que en años posteriores se produjo una mayor degradación de las condiciones laborales y salariales, lo que intensificaría los patrones de reproducción socioespacial de los grupos más pobres y dependientes, al acrecentar su necesidad de apoyo y limitar la movilidad. Sería de interés comprobar si la mejora de la situación en términos macroeconómicos de los últimos años ha alcanzado a reducir la dependencia de las redes familiares o si tal crecimiento agregado no ha supuesto una diferencia efectiva en la situación de muchos hogares. De ahí la necesidad de continuar este tipo de trabajos contando con datos más completos y actualizados, una preocupación que no es meramente académica, sino imprescindible para incorporarlos al debate y a la agenda política.

\section{Metodología}

\section{Fuente de datos y selección de la muestra}

Los datos en los que se basa este artículo provienen de la Encuesta de Población y Vivienda realizada por el Instituto de Desarrollo Regional de la Universidad de Granada y encargada por el Ayuntamiento de la ciudad en el año 2008. Realizada para prever las demandas de vivienda futura en el área metropolitana, la encuesta es una base de datos especialmente adecuada para el estudio de cuestiones residenciales. Disponemos de información relativa a trayectorias de movilidad en los últimos 10 años, convivencia y vida cotidiana en el barrio, expectativas de movilidad futura, etc. En lo que respecta a los objetivos de este artículo, la encuesta proporciona información relativa a las raíces familiares en el barrio y a la localización y extensión espacial de la red familiar (familiares directos — padres, hermanos, hijos- así como otros miembros de la familia exten- 
sa). En España no existen fuentes de datos que contengan dicha información combinada con datos exhaustivos de movilidad, por lo que los resultados de nuestro estudio podrán ser modestos (en tanto que referidos a un caso), pero permiten acercarse a una realidad escasamente analizada en nuestro país.

Se empleó un muestreo bietápico estratificado que cumplía unas condiciones impuestas de antemano de cuotas de edad y sexo; por ello, la primera unidad de muestreo era la sección censal y la segunda era la vivienda dentro de la sección censal. Las secciones censales se seleccionaron con probabilidad proporcional al número de viviendas en la sección. Sin embargo, con objeto de hacer más preciso el muestreo, las secciones censales se consideraron agrupadas en unos estratos que resumían las características socioeconómicas de los mismos, pues estas podían influir en los resultados de la encuesta. Tales estratos fueron construidos a partir de un análisis de conglomerados con datos censales del año 2001 de los municipios de interés para nuestro estudio. La muestra final, representativa de la población del área metropolitana de Granada, fue de 2363 entrevistas, todas ellas realizadas a individuos mayores de 18 años.

De los 2363 individuos, descartamos aquellos que aún viven en el hogar de sus padres. Incluir a los jóvenes no emancipados implicaría errores en la medición de su red familiar fuera del hogar y reflejaría una conducta residencial que no es resultado de sus decisiones, sino de las de sus progenitores. Por otra parte, descartamos a todos los individuos que no vivían en el área metropolitana antes de su último cambio de domicilio, esto es, a los inmigrantes que llegaron a Granada - procedentes de otros lugares de España o del extranjero-. Esta exclusión se debe a la nula probabilidad que tienen dichos grupos de haber realizado un cambio de domicilio dentro del área metropolitana (dado que no se encontraban residiendo en la misma). Sin embargo, todos los individuos de otras procedencias que llegaron a Granada antes del último cambio de domicilio registrado por la encuesta sí se encuentran incluidos en nuestro estudio. Tras esta selección de casos, la muestra finalmente incluida la componen 2090 individuos.

\section{Operacionalización de las variables depen- dientes}

En primer lugar, nos interesa conocer quién vive cerca de su familia por lo que es imprescindible definir qué consideraremos "cerca" y a quién consideraremos cuando hablamos de "familia". En este estudio adoptamos el barrio como medida de la proximidad entre familiares. A este respecto, cabe destacar que son los entrevistados los que definen si viven en el mismo/otro barrio; no se pedía la ubicación exacta. Por tanto, el barrio no es un ámbito impuesto por el investigador sino concebido por el propio individuo, lo cual introduce cierta subjetividad, pero, al tiempo, re- fleja mucho mejor la dimensión percibida de la proximidad, que es la que realmente afecta a las conductas (De Pablos y Susino 2010). En cuanto a la familia, entendemos que la forman los parientes con los que se tiene relación frecuente. Previamente se elaboraron modelos que tenían en cuenta únicamente a familiares cercanos - padres y hermanos-y se comprobó que los resultados eran bastante similares.

La variable dependiente en la segunda parte del artículo, la (in)movilidad residencial, se ha construido como una variable con tres categorías, en función del ámbito en el que se realiza el movimiento. Hemos tenido en cuenta el ámbito del último cambio de domicilio. Diferenciamos entre inmóviles; móviles dentro del barrio, y móviles entre barrios o comunidades. Aunque disponemos de información más detallada sobre el origen espacial del cambio de domicilio, esta clasificación en tres categorías resulta apropiada para el estudio de la relación entre capital familiar e (in)movilidad cuando la información sobre dicho capital se localiza en el espacio y, más concretamente, en el barrio (Kleinhans 2009).

\section{Variables explicativas y de control}

Para crear la variable referida al grado de concentración familiar en el barrio se calcula primero la extensión de la red familiar en el área metropolitana. Se tiene en cuenta la presencia de los tres tipos de parentesco por los que se pregunta en la encuesta: padres-suegros, hijos-yernos, hermanos-cuñados. Se contabiliza si los individuos tienen ninguno; un solo tipo de familiar; dos o tres tipos de familiares, o los cuatro tipos presentes en el área metropolitana y, posteriormente, cuántos de ellos viven en el barrio.

La segunda variable por la que nos preguntamos, los orígenes familiares en el barrio, ha sido calculada con base en dos preguntas. Con la primera cuestión generamos una variable que expresa si el individuo o su pareja (en caso de tenerla) nacieron en el barrio. Con la segunda, extraemos las raíces intergeneracionales, si sus padres o suegros nacieron allí. Esta subdivisión, aunque relevante de cara a conocer el arraigo familiar en el barrio, resulta poco significativa en nuestro contexto de estudio. Aunque con la construcción de la variable del grado de concentración la colinealidad no era un asunto problemático, sí resulta serlo con la variable relativa a las raíces familiares. Ambas variables creadas (raíces personales y raíces intergeneracionales) presentan un grado de colinealidad alto (mayor de 10 en el vif test - variance inflation factors-). Aunque la fuerte correlación entre las variables es una información relevante en sí misma, pone en riesgo la consistencia de los resultados si se incluyen ambos tipos por separado. Por ello, decidimos usar una única variable resumen de ambas. Consideramos que los individuos tienen raíces familiares si ellos o sus progenitores han nacido en el barrio. 
Tabla 1. Descripción de las variables

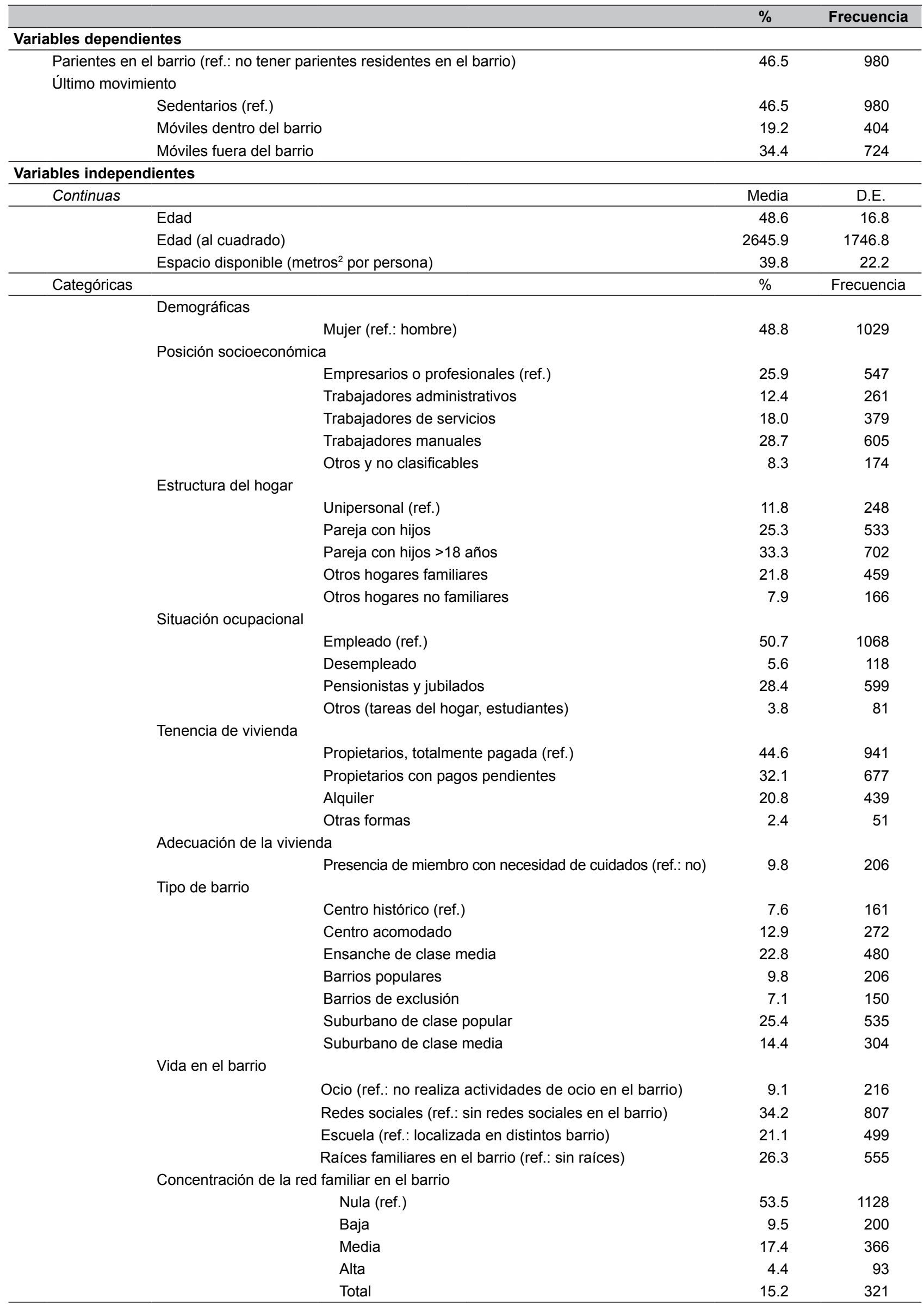

Fuente: Ayuntamiento de Granada. Encuesta sobre vivienda y población metropolitana. 2008 
En cuanto a las variables espaciales, el tamaño de la muestra no permite bajar a nivel de secciones censales, obligando a agrupar barrios demasiado amplios para tener sentido como tales. Por lo que hemos preferido clasificar las secciones por el nivel socioeconómico de su población en vez de por su ubicación, un procedimiento empleado en Susino y Palomares-Linares (2013). Con base en un análisis clúster con todas las secciones censales de Granada en 2001 empleando la variable "condición socioeconómica de los residentes", diferenciamos cinco zonas en la capital: casco histórico acomodado; centro acomodado; ensanches de clase media; barrios populares, y barrios desfavorecidos. La corona metropolitana la subdividimos en dos: municipios de clase media y municipios populares.

Con el fin de completar nuestros modelos, incluimos la edad, el sexo, la condición socioeconómica, el régimen de tenencia y la composición del hogar. Todos ellos son factores ampliamente reconocidos como predictores de las decisiones residenciales. La presencia de algún miembro en el hogar con necesidad de ayuda funcional ha sido también incluida, debido a su importancia para el estudio del papel de la familia en la movilidad. En cuanto a las variables relacionadas con el barrio, incluimos si se localizan allí sus actividades de tiempo libre, la escuela (en caso de tener hijos en edad escolar) o su red de amigos.

\section{Técnicas y procedimiento}

Para contestar nuestra primera pregunta de investigación —quiénes son más propensos a vivir cerca de sus familiares-, hemos realizado un análisis de regresión logística. Se han elaborado tres modelos sucesivos. El primero incluye solo las variables sociodemográficas individuales y del hogar. En el segundo modelo, agregamos la clasificación de zonas urbanas. En el tercero, también incluimos una variable de (in)movilidad espacial que tiene en cuenta el ámbito en el que se produce el último cambio de residencia. Al agregar estas dos variables por separado, contrastamos su relación con la variable dependiente y obtenemos un primer indicio de la conexión existente entre familia, espacio urbano y movilidad.

Para analizar la relación entre nuestras variables familiares explicativas - raíces y concentración familiar en el barrio-y la (in)movilidad espacial, realizamos un análisis de regresión logística multinomial. Para comprobar en qué medida la presencia de raíces y el grado de concentración mejoran la calidad de la estimación, las incluimos por separado y observamos cómo varían las medidas de ajuste del modelo. Aunque en este documento solo presentamos el modelo final, los resultados de dicha comprobación son comentados más adelante y pueden ser solicitados a los autores.
Antes de realizar ambos análisis, hemos comprobado la existencia de multicolinealidad entre las variables incluidas, así como los posibles problemas de heterocedasticidad y falta de especificación. Aunque los resultados no indican problemas al respecto, los coeficientes referidos a la existencia de heterocedasticidad están cercanos al límite de aceptación, por lo que decidimos estimar los errores utilizando una función robusta. Para una más clara interpretación de los resultados de los modelos multinomiales, se han calculado y se presentan los efectos marginales medios (AME's) y no los coeficientes beta (B) o los riesgos relativos ( $R R$ ).

Por último, para conocer la posible interconexión de las variables explicativas familiares con variables sociales a la hora de predecir sus efectos en la (in) movilidad, hemos calculado interacciones entre nuestras variables clave - concentración y presencia de raíces familiares - sobre el tipo de zona urbana, así como tenido en cuenta la condición socioeconómica de los individuos. De esta forma, comprobamos si los efectos que puedan tener la localización y concentración de las redes familiares son más acusados en función del lugar donde se vive o de la clase social de pertenencia. En el caso de la interacción entre las variables familiares y la condición socioeconómica, y dado que el conocido "efecto barrio", presente, sobre todo, en barrios de exclusión, en los que la presencia de familiares puede ser una consecuencia de las constricciones a la hora de salir de esas zonas (van Ham et al. 2014), se tiene en cuenta en la primera interacción —variables familiares-tipo de zona-, decidimos excluir a los residentes en zonas deprimidas, ya que podrían sesgar los resultados.

\section{¿QUIÉN VIVE CERCA DE SU FAMILIA EN GRANADA?}

En nuestro análisis hemos encontrado algunas discrepancias con el contexto internacional acerca de quiénes viven en el mismo barrio que sus parientes (tabla 2). La probabilidad de vivir cerca de un familiar pierde su relevancia cuando controlamos el tipo de área urbana en que viven los encuestados. Aunque otros estudios han detectado diferencias basadas en el sexo o el momento del curso vital, estas diferencias no parecen estadísticamente relevantes para el caso de Granada. Pero este dato debe ser tratado con cuidado: se apunta que no existen grandes diferencias en la importancia de la familia entre los entrevistados, por lo que también podríamos interpretar que es igualmente importante para todos. El resto de variables significativas no se ven alteradas por la inclusión de nuevos factores en los modelos 2 y 3 , lo que confirma que se trata de variables relevantes y no solapadas con las anteriormente incluidas. Los estadísticos de ajuste confirman que cada uno de los modelos supone una mejora con respecto al anterior. 
Tal como esperábamos y en concordancia con la literatura internacional, hay ciertas características asociadas más intensamente con la probabilidad de vivir cerca de la familia. Por ejemplo, los hogares donde algún integrante necesita cuidados. En relación con otros estudios se deduce que este patrón es universal, al menos en países desarrollados. Y es plausible que su capacidad predictiva sea inclu- so mayor en España, dado que las redes familiares son las encargadas de llevar el peso principal de la provisión de servicios asistenciales. Pese a que las opiniones acerca de sobre quién deberían caer estas responsabilidades están cambiando, gran parte de la población sigue considerando que la familia debe ser la principal responsable de ayudar a sus dependientes, incluso si la administración pública asumiera la

Tabla 2. Modelo logit sobre la proximidad con la familia (ref.: $\sin$ familiares en el barrio)

\begin{tabular}{|c|c|c|c|c|c|c|}
\hline \multirow[b]{2}{*}{ Demográficas } & \multicolumn{2}{|c|}{ Modelo 1} & \multicolumn{2}{|l|}{ Modelo 2} & \multicolumn{2}{|l|}{ Modelo 3} \\
\hline & Coef. & EE & Coef. & EE & Coef. & EE \\
\hline Edad & -0.012 & 0.019 & -0.012 & 0.019 & -0.014 & 0.020 \\
\hline Edad (al cuadrado) & 0.000 & 0.000 & 0.000 & 0.000 & 0.000 & 0.000 \\
\hline Mujer & -0.039 & 0.119 & -0.013 & 0.120 & -0.050 & 0.124 \\
\hline \multicolumn{7}{|c|}{ Posición socioeconómica (ref.: empresarios o profesionales) } \\
\hline Trabajadores administrativos & 0.518 ** & 0.158 & $0.432 * *$ & 0.160 & $0.384 *$ & 0.165 \\
\hline Trabajadores de servicios & $0.567^{* * *}$ & 0.143 & 0.420 ** & 0.147 & $0.415^{* *}$ & 0.150 \\
\hline Trabajadores manuales & $0.913^{* * *}$ & 0.130 & 0.642 *** & 0.138 & 0.514 *** & 0.143 \\
\hline Autónomos & $0.845^{* * *}$ & 0.197 & $0.724^{* * *}$ & 0.202 & 0.672 ** & 0.208 \\
\hline Otros y no clasificables & 0.415 & 0.225 & 0.303 & 0.229 & 0.353 & 0.233 \\
\hline \multicolumn{7}{|l|}{ Estructura del hogar (ref.: hogar unipersonal) } \\
\hline Pareja con hijos & 0.483 * & 0.220 & 0.367 & 0.226 & 0.396 & 0.232 \\
\hline Pareja con hijos $>18$ años & 0.682 * & 0.283 & 0.477 & 0.292 & 0.509 & 0.301 \\
\hline Otros hogares familiares & 0.495 & 0.272 & 0.349 & 0.279 & 0.347 & 0.288 \\
\hline Otros hogares no familiares & -0.159 & 0.303 & -0.241 & 0.311 & -0.231 & 0.320 \\
\hline \multicolumn{7}{|l|}{ Situación ocupacional (ref.: ocupado) } \\
\hline Desempleado & 0.337 & 0.210 & 0.311 & 0.215 & 0.231 & 0.139 \\
\hline Pensionistas y jubilados & -0.009 & 0.154 & 0.042 & 0.157 & 0.028 & 0.168 \\
\hline Otros (tareas del hogar, estudiantes) & -0.442 & 0.329 & -0.343 & 0.334 & -0.346 & 0.328 \\
\hline \multicolumn{7}{|c|}{ Tenencia de vivienda (ref.: propietarios sin pagos pendientes) } \\
\hline Propietarios con pagos pendientes & $-0.310 *$ & 0.122 & $-0.390 * *$ & 0.125 & $-0.363 * *$ & 0.226 \\
\hline Alquiler & $-0.989 * * *$ & 0.151 & $-0.884^{* * *}$ & 0.154 & $-0.857^{* * *}$ & 0.160 \\
\hline Otras formas & 0.360 & 0.311 & 0.400 & 0.317 & 0.425 & 0.340 \\
\hline \multicolumn{7}{|l|}{ Adecuación de la vivienda } \\
\hline Espacio disponible & 0.005 & 0.004 & 0.003 & 0.004 & 0.003 & 0.004 \\
\hline Un miembro necesita cuidados (ref.: no nec.) & 0.401 * & 0.165 & 0.366 * & 0.167 & 0.433 * & 0.170 \\
\hline \multicolumn{7}{|l|}{ Tipo de barrio (ref.: casco histórico acomodado) } \\
\hline Centro acomodado & & & 0.163 & 0.235 & 0.174 & 0.238 \\
\hline Ensanche de clase media & & & 0.387 & 0.215 & 0.406 & 0.219 \\
\hline Barrios populares & & & $1.180^{* * *}$ & 0.250 & $1.275^{* * *}$ & 0.265 \\
\hline Barrios de exclusión & & & 0.696 ** & 0.264 & $0.763 * *$ & 0.270 \\
\hline Suburbano de clase popular & & & 0.742 *** & 0.217 & 0.758 *** & 0.225 \\
\hline Suburbano de clase media & & & $0.888^{* * *}$ & 0.231 & 0.896 *** & 0.239 \\
\hline \multicolumn{7}{|l|}{ Ámbito del último movimiento (ref.: sedentarios) } \\
\hline Mismo barrio de Granada & & & & & $0.750^{* * *}$ & 0.196 \\
\hline Otro barrio de Granada & & & & & -0.104 & 0.184 \\
\hline Mismo barrio del área metropolitana & & & & & $1.070 * * *$ & 0.247 \\
\hline Otro barrio del área metropolitana & & & & & -0.307 & 0.259 \\
\hline Suburbanización & & & & & -0.474 & 0.246 \\
\hline Otros ámbitos & & & & & $-0.597^{* *}$ & 0.229 \\
\hline Constante & -0.691 & & -1.011 & & -0.904 & \\
\hline $\mathrm{N}$ & 2108 & & 2108 & & 2108 & \\
\hline Log-ver. Solo constante & -1446.6 & & -1446.6 & & -1446.6 & \\
\hline Log-ver. Modelo completo & -1350.0 & & -1329.3 & & -1279.3 & \\
\hline $\mathrm{Chi}^{2}$ & 193.3 & & 234.4 & & 334.7 & \\
\hline Pseudo- $R^{2}$ & 0.067 & & 0.081 & & 0.116 & \\
\hline AIC & 2742.0 & & 2712.9 & & 2626.5 & \\
\hline
\end{tabular}

${ }^{* * *} p<0.001^{* *} p<0.01^{*} p<0.05$ EE: error estándar

Fuente: Ayuntamiento de Granada. Encuesta sobre vivienda y población metropolitana. 2008 
corresponsabilidad. Tener pagos pendientes o vivir de alquiler, son circunstancias que se asocian con una menor probabilidad de vivir cerca de la familia. Cuanto más estable es la forma de tenencia, más probable es la proximidad a la familia. Encontramos una asociación fuerte y positiva con las variables socioeconómicas, tanto individuales como de barrio. Esta relación parece tener un patrón claro: mientras más baja es la posición social, mayor probabilidad de vivir cerca de la familia.

La asociación es también positiva para algunos tipos de desplazamientos. Haberse movido dentro del mismo barrio se asocia con una mayor probabilidad de vivir cerca de parientes (al compararlos con los sedentarios). Este dato refuerza nuestra idea de la importancia del barrio para el estudio de las relaciones entre familia e (in)movilidad.

\section{RAÍCES, CONCENTRACIÓN DE LA RED FAMILIAR Y MOVILIDAD ESPACIAL}

El modelo multinomial (tabla 3) es coherente con la literatura, pero también aporta cuestiones más novedosas, como que el efecto de las variables es diferente entre las tres categorías de movilidad. La edad aumenta las probabilidades de ser sedentario, como también lo hace tener hijos mayores de 18 años en el hogar. Como esperábamos, la tenencia es crucial para explicar la movilidad. La posición socioeconómica no genera grandes diferencias en el sedentarismo, pero en cambio sí afecta a los patrones de movilidad. En Granada, en concordancia con trabajos anteriores (Fors y Lennartsson 2008), moverse dentro del barrio es más probable para los trabajadores manuales que para el resto de la población, lo que posiblemente tiene que ver con las dificultades para escoger un lugar diferente. Este efecto diferencial del estatus social desaparece al fijarnos en la movilidad fuera del barrio. La edad y la edad al cuadrado se vuelven significativas, pero la condición socioeconómica deja de serlo. El estatus social, útil para explicar otros procesos residenciales como la suburbanización (Susino y Duque-Calvache 2013), tiene escasa capacidad explicativa para analizar las diferencias en movilidad intraurbana. La escasez de datos sobre cambios dentro del municipio dificulta mucho la investigación de este tipo de movimientos, aunque existen algunos ejemplos recientes e interesantes en esa línea (López-Gay 2016).

Incorporar variables de control sobre el barrio mejora el ajuste general del modelo, lo que indica que el espacio de vida cotidiano está relacionado con las decisiones residenciales. Tener redes sociales en el barrio aumenta la probabilidad de quedarse y de moverse cerca. Pero no es tan importante para los que acuden al barrio procedentes de otras partes del área metropolitana. Por contraste, la situación del colegio de los hijos ejerce como ancla para los que se mue- ven dentro del barrio (como afirma Smits, 2010), pero es también un factor que puede incentivar la movilidad, buscando un mejor centro. La inclusión de las raíces familiares y la concentración familiar no afectan excesivamente al resto de parámetros y contribuyen a mejorar el ajuste del modelo. Por tanto, podemos concluir que estas variables tienen un papel en las decisiones acerca de los cambios de vivienda.

En conexión con nuestras preguntas de investigación e hipótesis, hemos detectado algunos efectos destacables de las variables familiares en la (in) movilidad residencial. Tener raíces familiares en el barrio tiene una conexión positiva y significativa con el sedentarismo y también con la movilidad dentro del barrio, pero el efecto marginal medio es más alto en los sedentarios. Las raíces están negativamente conectadas con la movilidad fuera del barrio y este vínculo es también significativo. Aunque investigaciones anteriores han señalado que las raíces familiares pueden ser un factor de movilidad (Fischer y Malmberg 2001), en nuestro modelo no hemos podido verificarlo. Los que tienen familia en el área metropolitana, pero no en su barrio, son menos dados a retornar a su proximidad. Este hecho puede ser debido a dos factores: (i) el perfil mayoritario de la movilidad residencial — parejas jóvenes_-, que eligen por su situación familiar y no tanto por sus vínculos emocionales con el lugar. En ese sentido, buscar la proximidad del colegio, un cierto tipo de vivienda o incluso un estilo de vida suburbana parecen mejores predictores que las raíces familiares; (ii) el tamaño del área metropolitana en estudio, cuya extensión podría permitir un contacto habitual aun residiendo en distintos puntos de la ciudad y su corona.

La concentración familiar tiene efectos significativos sobre la movilidad dentro del barrio. Todas las categorías muestran una clara relación y esta tiene una apariencia lineal. Mientras más concentrada espacialmente la familia, mayor movilidad de corto recorrido. De hecho, este es el parámetro que mejor predice tal cambio de domicilio. Por supuesto, no sabemos explícitamente el porqué de esta decisión de quedarse cerca de la familia — si se debe a motivos prácticos, emocionales, ausencia de alternativas...-, pero sí podemos concluir que tener familia en la proximidad actúa como un factor de estabilización. La localización del colegio y otras redes sociales en el barrio también son estadísticamente significativas y con influencia positiva, lo que concuerda con la idea de Dawkins (2006) de que la movilidad intrabarrio aumenta cuanto más diversos y profundos son los lazos que nos unen a él.

\section{VÍNCULOS FAMILIARES Y CLASE SOCIAL}

Pero, ¿son los efectos de las raíces y de la concentración de redes familiares diferentes en función del tipo de barrio? ¿Y al comparar el estatus 
Tabla 3. Modelo multinomial sobre movilidad espacial (ref.: sedentarios): efectos marginales medios (AME)

\begin{tabular}{|c|c|c|c|c|c|c|}
\hline & \multicolumn{2}{|c|}{ Sedentarios } & \multicolumn{2}{|c|}{ Móviles dentro del barrio } & \multicolumn{2}{|c|}{ Móviles fuera del barrio } \\
\hline & $\mathrm{dy} / \mathrm{dx}$ & DE & $\mathrm{dy} / \mathrm{dx}$ & DE & $\mathrm{dy} / \mathrm{dx}$ & DE \\
\hline \multicolumn{7}{|l|}{ Demográficas } \\
\hline Edad & $0.021^{* * *}$ & 0.004 & -0.005 & 0.003 & $-0.017^{* * *}$ & 0.004 \\
\hline Edad (al cuadrado) & $0.000^{* * *}$ & 0.000 & 0.000 & 0.000 & $0.000^{* *}$ & 0.000 \\
\hline Mujer & 0.033 & 0.021 & 0.021 & 0.020 & $-0.054 *$ & 0.022 \\
\hline \multicolumn{7}{|c|}{ Posición socioeconómica (ref.: Profesionales) } \\
\hline Trabajadores administrativos & -0.002 & 0.023 & 0.004 & 0.022 & -0.002 & 0.024 \\
\hline Trabajadores de servicios & -0.006 & 0.021 & $0.053 * *$ & 0.021 & -0.048 & 0.022 \\
\hline Trabajadores manuales & 0.014 & 0.033 & 0.025 & 0.032 & -0.039 & 0.037 \\
\hline Autónomos & 0.035 & 0.035 & -0.077 & 0.041 & 0.042 & 0.038 \\
\hline \multicolumn{7}{|c|}{ Estructura del hogar (ref.: hogares unipersonales) } \\
\hline Pareja con hijos & -0.005 & 0.031 & 0.007 & 0.029 & -0.003 & 0.033 \\
\hline Pareja con hijos $>18$ años & $0.092 * *$ & 0.034 & -0.057 & 0.032 & -0.034 & 0.034 \\
\hline Otros hogares familiares & 0.122 *** & 0.032 & -0.058 & 0.033 & -0.064 & 0.035 \\
\hline Otros hogares no familiares & 0.064 & 0.048 & -0.030 & 0.039 & -0.034 & 0.042 \\
\hline \multicolumn{7}{|c|}{ Tenencia de vivienda (ref.: propietarios sin pagos pendientes) } \\
\hline Propietarios con pagos pendientes & $-0.274 * * *$ & 0.016 & $0.123^{* * *}$ & 0.019 & $0.151^{* * *}$ & 0.021 \\
\hline Alquiler & $-0.249 * * *$ & 0.021 & $0.107^{* * *}$ & 0.024 & $0.143^{* * *}$ & 0.025 \\
\hline Otras formas & -0.067 & 0.054 & 0.019 & 0.050 & 0.048 & 0.062 \\
\hline \multicolumn{7}{|l|}{ Adecuación de la vivienda } \\
\hline $\begin{array}{l}\text { Un miembro necesita cuidados } \\
\text { (ref.: no nec.) }\end{array}$ & -0.062 * & 0.030 & -0.012 & 0.034 & 0.074 * & 0.035 \\
\hline \multicolumn{7}{|c|}{ Vida en el barrio (ref.: no localizado en el barrio) } \\
\hline Ocio & -0.042 & 0.020 & 0.024 & 0.019 & 0.019 & 0.021 \\
\hline Redes sociales & $0.070^{* * *}$ & 0.019 & $0.075^{* * *}$ & 0.016 & $-0.145^{* * *}$ & 0.020 \\
\hline Escuela & $-0.070 * *$ & 0.022 & 0.048 * & 0.023 & 0.022 * & 0.024 \\
\hline \multicolumn{7}{|c|}{ Concentración de la red familiar en el barrio (ref.: nula, ningún familiar en el barrio) } \\
\hline Baja & $-0.060 *$ & 0.029 & 0.073 * & 0.030 & -0.013 & 0.034 \\
\hline Media & -0.019 & 0.024 & $0.062 * *$ & 0.024 & -0.044 & 0.026 \\
\hline Alta & -0.033 & 0.048 & $0.101 *$ & 0.046 & -0.068 & 0.053 \\
\hline Completa & -0.042 & 0.025 & $0.126^{* * *}$ & 0.026 & $-0.085^{* *}$ & 0.029 \\
\hline \multicolumn{7}{|c|}{ Raíces familiares (ref.: sin raíces en el barrio) } \\
\hline Origen propio/de los padres & $0.108^{* * *}$ & 0.022 & $0.078^{* * *}$ & 0.022 & $-0.185^{* * *}$ & 0.022 \\
\hline $\mathrm{N}$ & 2108 & & & & & \\
\hline Log-ver. Solo constante & -2191.8 & & & & & \\
\hline Log-ver. Modelo completo & -1535.4 & & & & & \\
\hline $\mathrm{R}^{2}$ de McFadden & 0.299 & & & & & \\
\hline AIC & 1.534 & & & & & \\
\hline
\end{tabular}

Fuente: Ayuntamiento de Granada. Encuesta sobre vivienda y población metropolitana. 2008

socioeconómico de los individuos? Tal como se mantenía en la hipótesis de partida, los efectos son desiguales. En la figura 1 se muestra la influencia de las variables familiares en función de las zonas urbanas. Las diferencias no son especialmente significativas para los sedentarios, por lo que no se muestra el gráfico. Pero, para los que se desplazan dentro del barrio, ambas variables familiares son positivas, aunque con claras diferencias. La alta concentración familiar y las raíces son razones importantes para permanecer en el barrio, no solamente en los barrios de menor estatus, aunque el efecto es más marcado en las zonas populares y de exclusión.

Tal como esperábamos, nuestros datos demuestran que moverse dentro del barrio se relaciona con la reproducción social de las familias en el espacio. Es un proceso que ocurre en toda la ciudad de Granada, pero que afecta más a las familias en situación desaventajada. Si nos fijamos en los predictores sociales para los grupos que viven fuera de los barrios populares y de exclusión (figura 2), el papel de la concentración familiar sigue siendo más importante para los trabajadores manuales que para el resto de la población. Los trabajadores manuales son más propensos a moverse dentro del barrio cuando existe una alta concentración familiar en ellos, incluso si viven en zonas más acomodadas. Esto subraya la importancia de la familia como un recurso para aquellas personas con menos medios y la importancia, por tanto, de permanecer cerca de ella (incluso a costa de incurrir en mayores gastos de vivienda). 
Figura 1. Efectos marginales medios (AME's) de las variables familiares en la probabilidad de moverse en el barrio (izq.) y entre barrios (der.), en función del tipo de barrio

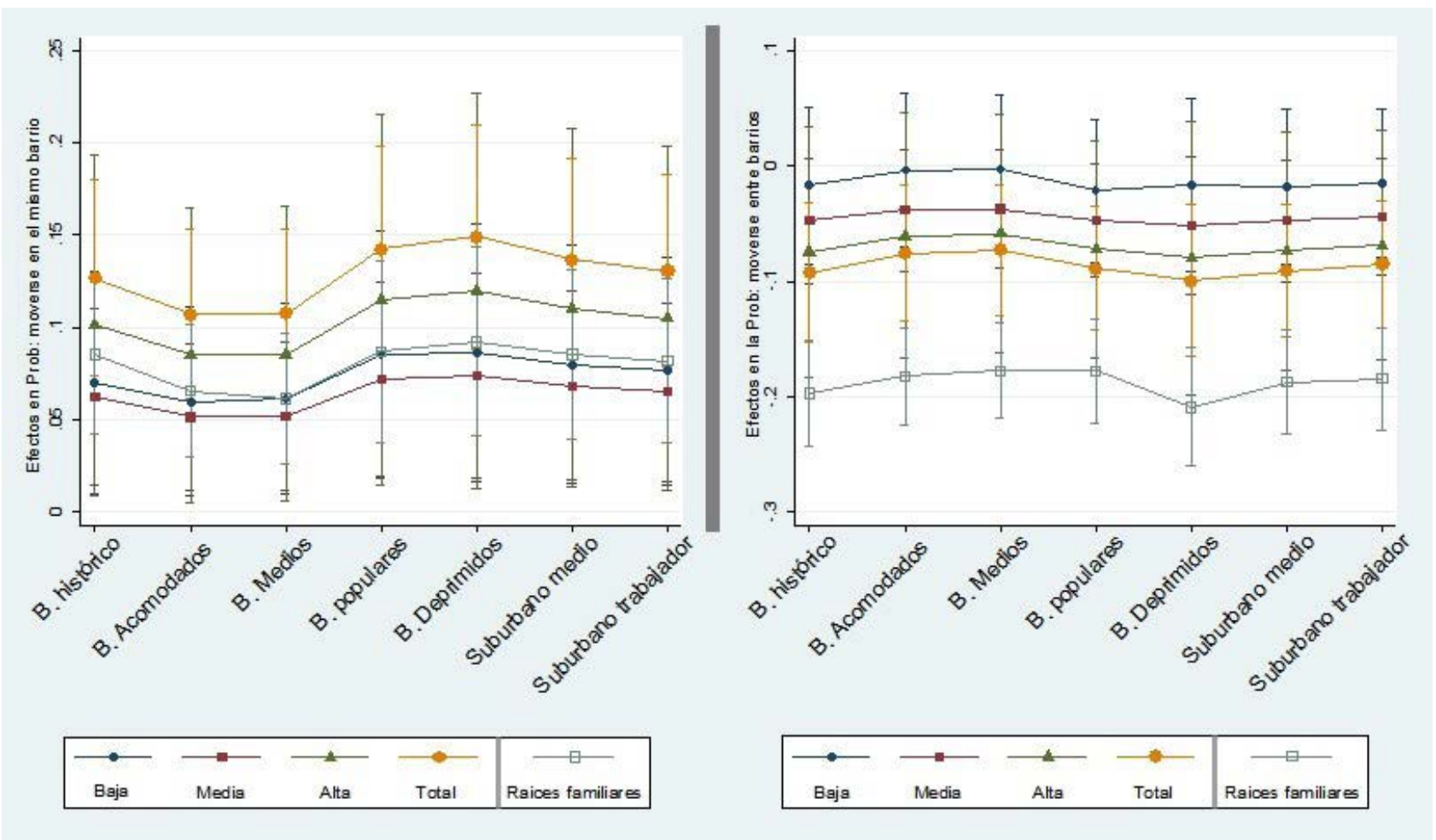

${ }^{*}$ AME's $95 \%$ Cls

** Se representa el eje Y de cada gráfico en diferente escala para visualizar de manera precisa los valores de AME's en cada categoría de la variable dependiente. Fuente: Ayuntamiento de Granada. Encuesta sobre vivienda y población metropolitana. 2008

Figura 2. Efectos marginales medios (AME's) de las variables familiares en la probabilidad de moverse en el barrio según la condición socioeconómica (excluyendo residentes en barrios deprimidos)

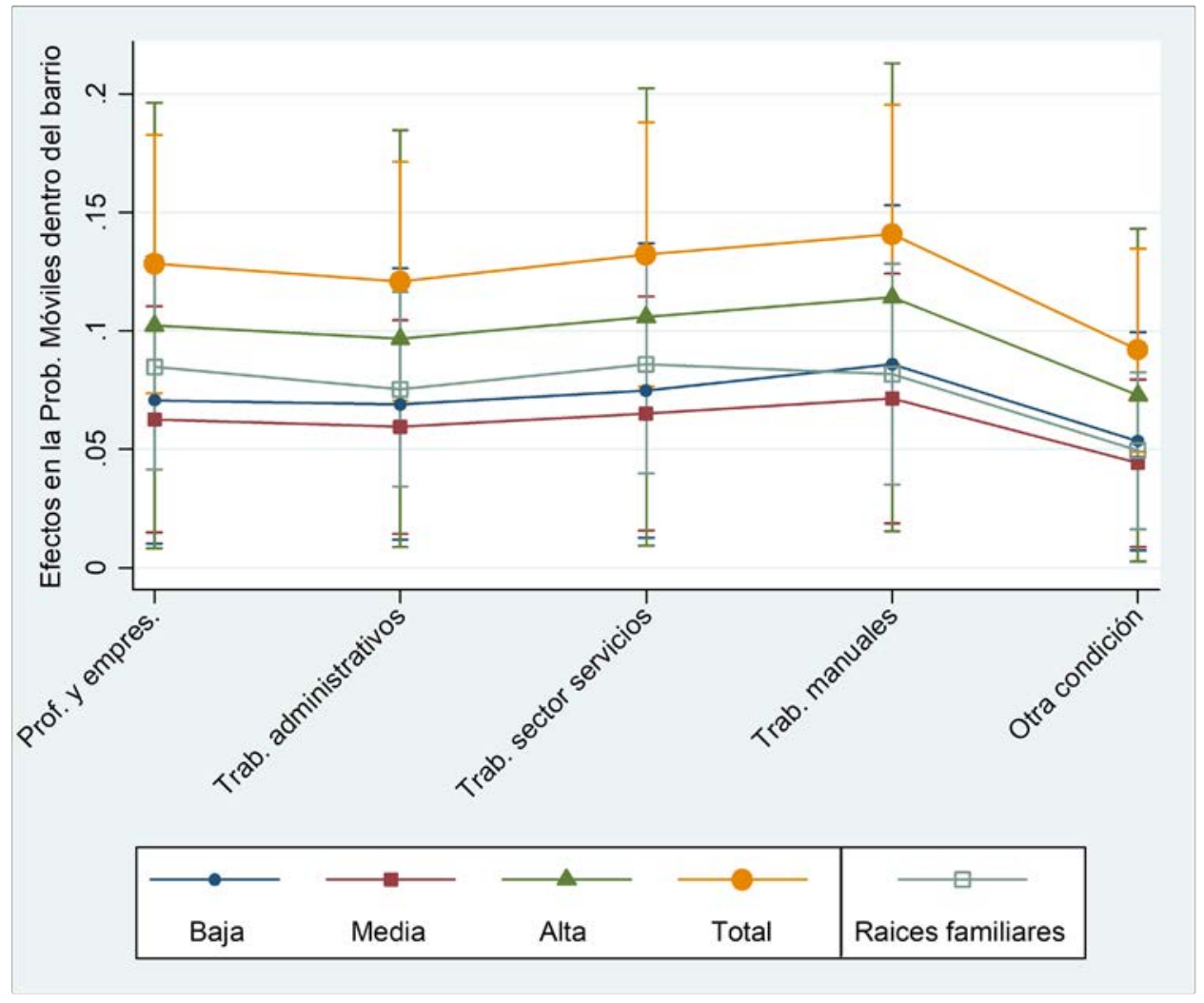

* AME's 95\% Cls

Fuente: Ayuntamiento de Granada. Encuesta sobre vivienda y población metropolitana. 2008 


\section{Conclusiones}

En este artículo hemos mostrado cómo, al estudiar una ciudad metropolitana mediana española, el papel de la familia en la (in)movilidad residencial tiene características similares a las encontradas en otros estudios, pero también patrones no descritos en trabajos previos realizados en otros países. La relación entre familia y residencia parece, pues, mediada por tendencias propias y relativas a la cultura y dimensión de la ciudad analizada. Pero también se comprueba que dicha relación entre elecciones residenciales y localización familiar parece ser una relación crucial no solo para aquellos que migran internacionalmente, sino también para las personas que realizan movimientos de corto recorrido.

En cuanto a la primera hipótesis planteada, parte de un planteamiento estático, una foto fija: ¿quién vivía cerca de su familia en la Granada de 2008? Nuestro modelo muestra que los factores explicativos son una mezcla de cuestiones transversales a toda la población (como tener un familiar dependiente, el modo de tenencia y la movilidad reciente) junto con factores asociados a la posición social (tanto del hogar como del barrio), donde los más desfavorecidos tienden a una mayor proximidad.

En cuanto a la segunda hipótesis, incorpora un elemento dinámico, al medir la relevancia de la familia en las decisiones de movilidad. La presencia de familia no es solo un resultado de la movilidad previa, sino un importante condicionante de nuevas conductas. Mientras la movilidad entre barrios se explica, en su mayor parte, por la variables habituales en estudios de movilidad, las variables de familia y vida cotidiana en el barrio son cruciales para explicar los movimientos intrabarrio $-\mathrm{y}$, en menor medida, el sedentarismo-. De manera que la familia se convierte en un elemento esencial para entender la reproducción social en espacios urbanos. Este dato es relevante de cara a entender las resistencias a las intervenciones que intentan romper dinámicas de exclusión mediante la relocalización de familias: moverse fuera implica grandes costes, no solo emocionales sino también a nivel de recursos. Esta idea nos permite enlazar la reflexión con el contexto económico: posiblemente la crisis ha acentuado la relevancia del apoyo familiar desde 2008. Pero depender de esa ayuda dificulta la movilidad espacial y social (Palomares-Linares y van Ham 2018), lo que a su vez puede limitar las opciones de mejora de la situación económica familiar.

Podría ser interesante redefinir la cuestión que plantea Dawkins (2006) sobre las redes sociales: ¿están las redes familiares atando a las personas a los lugares? Según nuestros datos, lo hacen. La proximidad con los parientes podría ser descrita como parte de un ciclo de reproducción social al que es difícil asignar un comienzo o un fin. No podemos estar seguros de si la gente se queda porque tiene familia cercana, de forma estratégica (Preece 2018), o si, más bien, tiene a la familia tan próxima por no haberse podido mover.

Nuestra tercera hipótesis es que la familia afecta a la movilidad residencial de manera diferente en función de la clase social. Independientemente de las características del hogar o del individuo concreto, todos los granadinos tienden a permanecer en el barrio si tienen parientes que viven cerca. En contextos como el de nuestro estudio, la familia importa, sin importar qué tipo de familia consideremos. Pero, dentro de esta relevancia, existen diferencias notorias en significados y funcionalidades. Tal como Bell y Rutherford (2013) apuntan, la red familiar se estructura en niveles y, posiblemente, cada nivel aporta diferentes formas de apoyo. Cuando las necesidades no se limitan a lo emocional, la concentración de redes familiares se vuelve más importante. Es decir, cuando los miembros se encuentran en una posición desfavorecida y con mayor necesidad de recursos instrumentales buscan la proximidad de la familia con mayor probabilidad que aquellos que disponen de más recursos.

¿Hasta qué punto los resultados tienen que ver con las condiciones urbanas y demográficas de nuestro caso? Es complicado determinarlo, de ahí el interés de emprender estudios comparativos con otras áreas metropolitanas. De cara al futuro se pueden plantear nuevas cuestiones de interés. Por ejemplo, cuando las distancias urbanas se amplían, ¿lo hacen también las redes familiares? ¿Impiden los precios en los mercados inmobiliarios más activos la reproducción espacial de las familias? ¿O tal vez potencian la formación de zonas de exclusión, impulsando una reproducción constante de la segregación? En cualquier caso, consideramos fundamental incluir la dimensión familiar de una manera compleja y desagregada en los estudios venideros sobre comportamiento residencial, puesto que añade un nivel de significado primordial para entender las decisiones de individuos y familias.

\section{AgRADECIMIENTOS}

Isabel Palomares agradece la financiación de este trabajo a la Universidad de Granada, a través de un contrato puente obtenido en la convocatoria 2017 del Plan Propio de Investigación (resolución de 26-2-2018). Este trabajo forma parte de los resultados del proyecto de investigación "Procesos de reconfiguración social metropolitana" (CSO2014-55780-C3-3-P), del Plan Nacional de I+D. 


\section{REFERENCIAS BIBLIOGRÁFICAS}

Alabart, A. 2007. "Mobilitat residencial, solidaritat familiar i ciutadania a les regions metropolitanes", $R e-$ vista catalana de sociología 22: 23-39. https://doi. org/10.2436/20.3005.01.11

Bell, D. y A. Rutherford. 2013. "Individual and geographic factors in the formation of care networks in the UK", Population, Space and Place 19: 727-737. https://doi. org/10.1002/psp.1792

Blaauboer, M. 2011. "The impact of childhood experiences and family members outside the household on residential environment choices", Urban Studies, 48: 1635-1650. https://doi.org/10.1177/0042098010377473

Campbell, K. E., P. V. Marsden y J. S. HurlbertS. 1986. "Social resources and socioeconomic status, Social Networks 8: 97-117. https://doi.org/10.1016/S03788733(86)80017-X

Clark, R. y D. Wolf. 1992. "Proximity of children and elderly migration". Pp: 77-96 in Elderly migration and population redistribution: A comparative study, edited by A. Rogers, London, Belhaven.

Clark, W. A. V. y F. M. Dieleman. 1996. Households and housing. New Brunswick: NJ: CUPR Press.

Clark, W. A. V., R. Duque-Calvache e I. Palomares-Linares. 2015. "Place Attachment and the decision to stay in the neighbourhood", Population Space and Place 23(2), e2001. https://doi.org/10.1002/psp.2001

Cooke, T. J. 2008. "Migration in a family way", Population, Space and Place 14: 255-265. https://doi.org/10.1002/psp.500

Coulter R., M. Van Ham y A. M. Findlay. 2015. "Re-thinking residential mobility: linking lives through time and space", Progress in Human Geography 40(3): 352-374 https://doi.org/10.1177/0309132515575417

Crowder, K. y S. J. South. 2005. "Race, class, and changing patterns of migration between poor and nonpoor neighborhoods", American Journal of Sociology 110: 1715-1763. https://doi.org/10.1086/428686

Da Vanzo, J. 1981. "Repeat migration, information costs, and location-specific capital", Population and Environment 4(1): 45-73. https://doi.org/10.1007/BF01362575

Dawkins, C. J. 2006. "Are social networks the ties that bind families to neighborhoods?", Housing Studies 21: 867888. https://doi.org/10.1080/02673030600917776

Feijten P., P. Hooimeijer y C. H. Mulder. 2008. "Residential experience and residential environment choice over the life-course", Urban Studies 45: 141-162. https://doi. org/10.1177/0042098007085105

Feria, J. M. 2015. Definición y pautas generales de dinámica y organización espacial: Áreas metropolitanas andaluzas. Sevilla: Consejería de Fomento y Vivienda. Junta de Andalucía.

Fischer, C. S. 1982. To Dwell Among Friends. Chicago: University of Chicago Press.

Fischer, P. A. y G. Malmberg. 2001. "Settled people don't move: on life course and (im-)mobility in Sweden", International Journal of Population Geography 7: 357371. https://doi.org/10.1002/ijpg.230

Flaquer, L. 2004. "La articulación entre familia y el Estado de bienestar en los países de la Europa del sur". Papers: Revista de sociología 73: 27-58. https://doi. org/10.5565/rev/papers/v73n0.1105

Fors, S. y C. Lennartsson. 2008. "Social mobility, geographical proximity and intergenerational family contact in Sweden", Ageing y Society 28: 253-270. https://doi. org/10.1017/S0144686X07006617
Granovetter, M. S. 1973. "The strength of weak ties", American Journal of Sociology 78(6): 1360-1380. https://doi. org/10.1086/225469

Hank, K. 2007. "Proximity and contacts between older parents and their children: A European comparison", Journal of Marriage and Family 69: 157-173. https://doi. org/10.1111/j.1741-3737.2006.00351.x

Hedman, L. 2013. "Moving near Family? The Influence of extended family on neighbourhood choice in an intraurban context", Population, Space and Place 19(1), 32-45. https://doi.org/10.1002/psp.1703

Joseph, A. E. y B. C. Hallman. 1998. "Over the hill and far away: Distance as a barrier to the provision of assistance to elderly relatives", Social Science and Medicine 46(6): 631-639. https://doi.org/10.1016/S02779536(97)00181-0

Jordan, T. G. 1988. The European culture area. New York: Harper y Row.

Kleinhans, R., M. van der Land y W. Doff. 2010. "Dealing with living in poor neighbourhoods", Journal of Housing and the Built Environment 25(4): 381-389. https://doi. org/10.1007/s10901-010-9194-5

Kleinhans, R. 2009. "Does social capital affect residents' propensity to move from restructured neighbourhoods?", Housing Studies 24(5): 629-651.

Kolk, M. 2016. "A Life-Course Analysis of Geographical Distance to Siblings, Parents, and Grandparents in Sweden", Population, Space and Place 23 (3), e2020. https://doi.org/10.1002/psp.2020

Litwak, E. y J. Szelenyi. 1969. "Primary group structures and their functions: kin, neighbours, and friends", American Sociological Review 34: 465-481. https://doi. org/10.2307/2091957

López-Gay, A. 2016. "Atracción de talento y polarización socioeconómica en Barcelona", Perspectives Demogràfiques 3: 1-4.

Michielin, F., C. H. Mulder y A. Zorlu. 2008. "Distance to parents and geographical mobility", Population, Space and Place 14: 327-345. https://doi.org/10.1002/ psp. 509

Michielin, F. y C. H. Mulder. 2008. "Family events and the residential mobility of couples", Environment and Planning A 40: 2770-2790. https://doi.org/10.1068/a39374

Módenes Cabrerizo, J.A. 1998. "Flujos espaciales e itinerarios biográficos: La movilidad residencial en el área de Barcelona". Tesis doctoral. Departamento de Geografía. Universitat Autònoma de Barcelona.

Mulder, C.H. 2007. "The family context and residential choice: a challenge for new research", Population, Space and Place 13: 265-278. https://doi. org/10.1002/psp.456

Mulder, C. H. y T. J. Cooke. 2009. "Family ties and residential locations", Population, Space and Place 15: 299-304. https://doi.org/10.1002/psp.556

Mulder, C.H. y M. J. van der MeerJ. 2009. "Geographical distances and support from family members", Рopulation, Space and Place 15(4): 381-399. https://doi. org/10.1002/psp.557

Mulder, C.H. y G. Malmberg. 2014. "Local ties and family migration", Environment and Planning A 46: 2195-2211. https://doi.org/10.1068/a130160p

Pablos, J.C. de y J. Susino. 2010. "Vida Urbana: entre la desigualdad social y los espacios del habitar", Anduli 9: 119-142. 
Palomares-Linares, I. 2017. "Movilidad residencial y sedentarismo en contextos urbanos". Tesis doctoral. Departamento de Sociología. Universidad de Granada.

Palomares-Linares, I. y M. van Ham. 2018. "Understanding the effects of homeownership and regional unemployment levels on internal migration during the economic crisis in Spain", Regional Studies. Consulta 22 de Marzo 2019. https://doi.org/10.1080/00343404.2018. 1502420

Pettersson, A. y G. Malmberg. 2009. "Adult children and elderly parents as mobility attractions in Sweden", Population, Space and Place 15(4): 343-357. https://doi. org/10.1002/psp.558

Preece, J. 2018. "Immobility and insecure labour markets: An active response to precarious employment", Urban Studies 55: 1783-1799.

Puga, D. 2004. "El comportamiento residencial de los mayores. Análisis biográfico de la movilidad en la vejez", REIS 105(1): 79-102. https://doi. org/10.2307/40184625

Rainer, H. y T. Siedler. 2012. "Family location and caregiving patterns from an international perspective", Population and Development Review 38(2): 337-351. https://doi. org/10.1111/j.1728-4457.2012.00495.x

Reher, D. S. 1998. "Family ties in Western Europe. Persistent contrasts", Population and Development Review 24: 203-234. https://doi.org/10.2307/2807972

Rossi, A. S. y P. H. Rossi. 1990. Of Human Bonding: ParentChild Relations across the Life Course. New York: Aldine de Gruyter.

Smits, A. 2010. "Moving close to parents and adult children in the Netherlands: the influence of support needs", Demographic Research 22: 985-1014. https://doi. org/10.4054/DemRes.2010.22.31

Spilimbergo, A. y L. Ubeda. 2004. "Family attachment and the decision to move by race", Journal of Urban Economics 55: 478-497. https://doi.org/10.1016/j.jue.2003.07.004

Spring, A., E. Ackert, K. Crowder y S. J. South. 2017. "Influence of proximity to kin on residential mobility and destination choice: Examining local movers in metropolitan areas", Demography 54(4): 1277-1304.

Susino, J. 2003. "Movilidad residencial. Procesos demográficos, estrategias familiares y estructura social". Tesis doctoral. Departamento de Sociología. Universidad de Granada.
Susino J. y R. Duque-Calvache. 2013. "Veinte años de suburbanización en España (1981-2001). El perfil de sus protagonistas", Documents d'Anàlisi Geogràfica 59(2): 265-290. https://doi.org/10.5565/rev/dag.31

Susino J. e I. Palomares-Linares 2013. "La movilidad residencial en el área metropolitana de Granada”. Pp. 345363 en Desarrollo Regional Sostenible en tiempos de crisis, editado por J. A. Camacho Ballesta e Y. Jiménez Olivencia. Granada: Universidad de Granada.

Thomése, G. y T. Van Tilburg. 2000. "Neighbouring networks and environmental dependency: differential effects of neighbourhood characteristics on the relative size and composition of neighbour networks in The Netherlands", Ageing and Society 20: 55-78. https://doi. org/10.1017/S0144686X9900762X

Tomassini, C., S. Kalogirou, E. Grundy, T. Fokkema, P. Martikainen, M. Broese van Groenou y A. Karisto. 2004. "Contacts between elderly parents and their children in four European countries: current patterns and future prospects", European Journal of Ageing 1: 54-63. https://doi.org/10.1007/s10433-004-0003-4

Van Groenou, M., y T. Van Tilburg. 2003. "Network size and support in old age: differentials by socio-economic status in childhood and adulthood", Ageing and Society 23(5): 625-645. https://doi.org/10.1017/ S0144686X0300134X

van Ham, M. y P. Feijten. 2008. "Who wants to leave the neighbourhood? The effects of being different from the neighbourhood population on wishes to move", Environment and Planning A 40(5): 1151-1170. https://doi. org/10.1068/a39179

van Ham, M., L. Hedman, D. Manley, R. Coulter y J. Östh. 2014. "Intergenerational transmission of neighbourhood poverty: an analysis of neighbourhood histories of individuals", Transactions of the Institute of British Geographers 39: 402-417. https://doi.org/10.1111/ tran. 12040

Wellman, B. y S. D. Berkowitz. 1988. Social Structures: a network approach. Cambridge: Cambridge University Press.

Wood L. y B. Giles-Corti. 2008. "Is there a place for social capital in the psychology of health and place?", Journal of Environmental Psychology 28(2): 154-163. https://doi. org/10.1016/j.jenvp.2007.11.003

Zorlu, A. y J. Latten. 2009. "Ethnic sorting in the Netherlands", Urban Studies, 46: 1899-1923. https://doi. org/10.1177/0042098009106023

ISABEL PALOMARES LINARES es doctora en Sociología por la Universidad de Granada, actualmente contratada como investigadora en el Departamento de Sociología de esta misma Universidad. Sus temas principales de trabajo son en el sedentarismo y la movilidad espacial y la importancia de los factores familiares y de barrio en las decisiones residenciales.

RICARDO DUQUE CALVACHE es doctor en Sociología por la Universidad de Granada y profesor en el Departamento de Sociología de dicha institución. Sus temas de investigación giran en torno a la sociología urbana y la demografía, con especial interés en las áreas metropolitanas, la movilidad residencial y los procesos de gentrificación.

JOAQUÍN SUSINO ARBUCIAS es doctor en Sociología por la Universidad de Granada y profesor en el Departamento de Sociología de dicha institución. Especializado en el estudio y la docencia de la demografía, con especial interés en el estudio de las áreas metropolitanas y los procesos de transformación social vinculados a su existencia y desarrollo. 